\title{
CASE OF FRACTURE OF THE SKULL, WITH ESCAPE OF BRAIN-SUBSTANCE; HERNIA CEREBRI.
}

\author{
BY THOMAS EVELYN LITTLE, M.D., L.R.C.S.;
}

University Anatomist, and Surgeon to Sir Patrick Dun's Hospital.

[Read in the Section of Pathology, November 1, 1889.]

Tre case I wish to detail (the specimens of which I exhibit) is one of fracture of the skull, in a child three years of age. The accident occurred on August 11, 1889.

The mode in which the accident occurred was this:-The father and mother of the child had been quarrelling-as their manner was-and, as an incident in the fight, the difference of opinion having been renewed after the mother went to bed with the child sleeping beside her, the husband seized a wooden stool, and flung it violently at his wife. His aim not being very steady, for reasons which the hour of the night-it was one o'clock-rendered easily intelligible, and the room being badly lighted, the missile missed the mother, but struck the child on the right frontal region. The infant, when it was recognised to be injured, was immediately carried by another man, who was present, to Sir Patrick Dun's Hospital.

The condition of the patient on being brought to hospital was as follows:-A considerable wound was observed on the right frontal region, which was bleeding slightly, and from it some lacerated brain-substance had escaped, and was escaping - to such an extent, indeed, that even the sleeve of the coat of the man who had carried the infant, and upon whose arm the poor child's head had rested, was bespattered with portions of the brain. Notwithstanding this, the child presented no symptoms of collapse or of concussion of the brain. It was quite conscious—so much so, indeed, that it resisted, 
and fought with the house surgeon on his attempting to examine the wound.

When I first saw the patient-about eight hours after admission-on removing the dressings from the head, it presented a scalp wound in the situation just below the right frontal eminence of about three inches in length, which was slightly gaping, and was stripped upwards from the bone, the periosteum of which was separated. The wound was full of broken-up brain substance and blood, which had also insinuated itself underneath the scalp-flap. On gently probing the wound with the finger, the sharp edge of a fracture of the calvaria, which extended backwards under the scalp wound, could be detected, with some, but very insignificant, depression. The size we had pretty accurately mapped out as about that of a florin. As to whether this portion of bone was fixed, or completely separated, I could not then satisfy myself, by any amount of investigation which $I$ deemed to be justifiable; but it became subsequently obvious that it was fixed at the posterior part. The patient presented a total absence of any cerebral symptoms whatsoever. The child was quite observant and intelligent, and had not had, nor then had, any of the symptoms of cerebral concussion or cerebral irritation. There was no paralysis of any kind, or any muscular spasm; and sensation everywhere-as far as capable of being tested in so young a subject-was unimpaired.

Progress of the Case.-I do not intend to go through, in detail, the particulars, as recorded from day to day in the hospital case book, but shall just mention the main features which became developed in the case.

The patient lived to the evening of the 15th day, dying then after the supervention of a convulsive attack-due (as we had diagnosticated it to be) to the occurrence of a diffuse arachnitis.

The phenomena which had developed in the interim, and on each of which I shall say a word or two, were :-Further and continued escape of brain-substance; discharge of serous fluid from the wound; hernia cerebri-gradually growing; divergence of head to right (2nd week); condition of wound; and mode of death.

The escape of brain-matter continued for some days, appearing 
on the successively-removed dressings up to the fifth day, after which it was no more observed.

The discharge of serous fluid from the wound-fluid which, from its character and mode of occurrence, was obviously analogous to, and had the same source as that we are so familiar with as escaping from the ear in cases of fracture of the skull's base-was a feature in this case; and was remarkable in one particular-i.e., its intermittence; it was tolerably copious primarily, for about the first forty-eight hours after the injury, when it ceased. It then recurred, to a slight extent and for a short time, on the seventh day, when it again subsided. For the last two days of the child's life, however, it again appeared with extreme copiousness, deluging and soaking the pillows and bedclothes.

A hernia cerebri-the occurrence of which we had anticipated, from the small size and obliquity of the wound in the dura mater-commenced to make its appearance on the eighth day, and gradually grew in size up to the time of death, when it had assumed about the size of half a large walnut. On the morning of the evening of death a large part of this fungus separated while dressing the wound; and, having been preserved in spirit, Dr. R. G. Patteson, who kindly undertook the investigation of the specimen, informs me as the result of his examination:- "It proved to be merely a piece of broken-down brain substance and blood-clot mixed; no trace of membranes."

The symptom of a persistent spasmodic divergence of the head to the right side (that of the injury) which appeared, and was present during the last week of the child's life, is one which I think more especially worthy of note, as being the only solitary motor phenomenon developed during the progress of this case, up to the occurrence of the final and fatal convulsion. There was no associated deviation of the eyes, which were easily and voluntarily movable in all directions. Upon this symptom I shall make a very brief remark further on.

As to the condition of the wound, it appeared to assume readily a very healthy character, which it maintained throughout. There was no deep-seated suppuration, or bagging of pus-in fact no 
suppuration except at the very edges, which were healthily granulating, and cicatrising at the time of death. There was at no period any offensive odour; and, indeed, as far as the physical senses can be a test, the wound remained aseptic.

The mode of death was by the supervention of a severe and protracted convulsive attack, which, although apparently commencing by a twitching of the limbs of the left side, was afterwards much more marked on, and eventually confined to, the right side.

Post-mortem Examination.-(The calvaria, brain, and membranes were exhibited). On examining the body after death, we observed a condition of things, which is in chief part to be still seen in the specimens before the meeting.

The position, size, and condition of the scalp wound were as I have described them above.

The calvaria, with the dura mater attached, are here to be seen. Just at and below the right frontal eminence there is a fracture of the skull, of approximately circular shape, and of about the size of a half-crown piece, slightly depressed in front. Anteriorly and laterally, the bone is completely and sharply broken, but behind there is no fracture, or solution of continuity of bony tissue, the bone being here simply gradually bent inwards. A somewhat sharp point of the bone anteriorly, it can be seen, has penetrated and wounded the dura mater, a rent in which exists, of about half an inch in length, somewhat obliquely situated with regard to the opening in the bone. The depression of the bone was only to the extent of three sixteenths of an inch.

On examining the brain, we observe a deep excavation in the frontal lobe, corresponding in position and size to the skull fracture. It extends for the depth of fully an inch into the substance of the brain, is remarkably circumscribed, and is unassociated with any extent of laceration, or softening of the brain tissue in the neighbourhood. The surfaces of both cerebral hemispheres are covered with a layer of greenish-yellow lymph, beneath the arachnoid, following the course of the sulci over the sides, and even to a slight extent the base, of the brain. It is, curiously 
enough, somewhat more extensive and diffused over the left than over the right side.

Remarks.-This case suggests some very interesting and important points, with reference to the subject of the particular form of fracture of the skull of which it is an example.

In the first place, the extraordinary immunity from primary (and, indeed, often of even any subsequent) symptoms of a serious character after severe and extensive brain injury, enjoyed by children of a tender age, is markedly exemplified. I calculated that this child must have lost, from first to last, at least half an ounce of cerebral tissue; and yet up to the final and fatal convulsion, with the single exception I have mentioned-of the persistent deviation of the head to the side-there was not, during the whole course of the case, a single symptom of interference with brain function. Of course, too, the situation of the cerebral destruction-i.e., almost wholly situated in the frontal region, in front of the now recognised motor area of the brain cortex-must be taken into account. We all know that the fact of the occurrence of extensive brain lesion in the frontal region without serious symptoms is a very old observation-one, i.e., long antecedent to any of the modern definite ideas as to cerebral cortical localisation. But, in this individual case of mine, the situation of the lesion, taken in connection with the same exceptional and single motor phenomenon to which I have alluded (deviation of the head to the right) is in no small degree interesting and deserving of a few moments' attention to details.

It will be remembered that the recent observations of Ferrier, Horsley, and others, have located a small area of the cortex of the frontal lobe, occupying the posterior extremities of the superior and middle frontal convolutions, just in front of the ascending frontal convolution, as the focal centre of what is termed the "conjugate deviation of the head and eyes," to the same side. Now, if we compare the exact position of the lesion in my case with this localisation, we observe that it (the latter) is just at its anterior part encroached upon by the posterior edge of the site of the fracture. The explanation, however, of the reason why, 
in this case, we had not (as I have mentioned, and as we certainly had not) the associated divergence of the eyes, I must leave to those who are more familiar with the interpretation of the minutiæ of cerebral localisation than I pretend to be. It may be that this case may suggest that the anterior part of this region is concerned with the lateral movements of the head, and its posterior part with the associated eye movements.

With regard to the laceration and escape of brain substance, which occurred to such an extent in this case, there is, I think, one small point of some slight importance-I mean with regard to precise mechanism of the brain extravasation. We know that laceration of the brain may take place from injury in different ways-thus, eg., it may occur as the direct result of the blow fracturing the skull, driving in, and perhaps comminuting the bone; or it may occur from cerebral contre-coup, at the opposite pole of the brain cavity. In the present case there is, however, at first sight, an apparent difficulty in explaining the amount of brain destruction, and of brain actually lost. As you see, there is no competent depression, and no comminution, or driving inwards of the bone. It is-when we particularly examine the fracture, and the size of the opening into the brain cavity-scarcely conceivable that the amount of cerebral substance which is lost can have escaped primarily through such a minute, and such an oblique, opening in the skull and dura mater from any simple internal pressure. I think, then, that the probable interpretation lies in an appeal to the nature of the injury, the age of the patient, and the precise details of the pathological lesion. The subject is a young child, where the bones of the calvaria are notoriously thin and pliable; and looking again at the specimen before us, you will observe-as, indeed, $I$ have already pointed out-that the fragment of broken bone is bent in, and (as it were) hinged inwards (without being fractured) at its posterior part. Considering, then, the nature of the injury, and the force with which the blow must have been delivered, associated with the altogether disproportionate depth to which we observe the wound of the brain to have extended (especially when compared with the very slight amount of 
bone which remains depressed), is it not a probable-indeed I should say almost only-solution of the difficulty that this hinging inwards of the bone (as I have ventured to call it) may have taken place to a much greater extent at the moment of the blow, and that the elastic infantile bone immediately springing outwards may have lacerated, and (so to speak) dug out, or spooned out, the large amount of brain matter found in, and in the neighbourhood of, the wound; and which we can still see to be missing in the specimen? The amount of brain matter actually removed, with the very slight depression which existed, readily explains, also, another pointviz., the absence of symptoms, or rather the non-existence, of any brain pressure.

There are some interesting questions in reference to the treatment in this case, which might be discussed; but these I do not look upon as available, or suitable for discussion in this Section of the Academy. 